\title{
COMPACTIFICATION OF MINIMAL SUBMANIFOLDS OF HYPERBOLIC SPACE
}

\author{
GERALDO DE OLIVEIRA FILHO
}

In this paper we study the geometry of complete minimal submanifolds of hyperbolic space $\mathbb{H}^{n}$. Specifically, we are interested in $m$-dimensional submanifolds whose second fundamental form $\mathcal{A}$ satisfies $\int_{M}|\mathcal{A}|^{m}<\infty$ where $|\mathcal{A}|$ is the norm of $\mathcal{A}$.

To motivate this hypothesis we briefly outline the main results when the ambient space is $\mathbb{R}^{n}$. Osserman [15] and Chern-Osserman [3], showed that for a complete minimal immersion (cmi for short) $M^{2} \rightarrow \mathbb{R}^{n}$, with finite total curvature, it is possible to compactify $M$ by the Gauss map $g: M \rightarrow G_{n, 2}$ which maps $p \in M$ to the 2-plane $T_{p}(M)$. By the Weierstrass representation $g$ is a holomorphic curve in $G_{n, 2}$, viewed as the complex quadric $Q_{n-2}=$ $\left\{z_{1}^{2}+\cdots+z_{n}^{2}=0\right\}$ of the complex projective plane $\mathbb{C} P^{n-1}$. They showed that when the total curvature $C(M)=\int_{M} K$ is finite, $M$ is of finite conformal type, i.e., $M$ is conformally equivalent to a closed surface $\bar{M}$ with a finite number of points removed, and that $g$ extends holomorphically to $\bar{M}$. In particular this implies that the total curvature is quantified by $C(M)=2 \pi k, k$ an integer, and that $M$ is properly immersed.

For a cmi $M^{m} \hookrightarrow \mathbb{R}^{n}, m \geq 2$, Anderson [2] has obtained a generalization of the Chern-Osserman result. He proved that $|\mathcal{A}|(p)$ goes to 0 , as the distance $d\left(p, p_{0}\right)$ of $p$ to a fixed point $p_{0}$ goes to infinity. Using the fact that the class of minimal submanifolds is invariant by the homotheties of $\mathbb{R}^{n}$, he proved that $|\mathcal{A}|(p)=\mu(p) / d^{2}\left(p, p_{0}\right)$, where $\mu(p) \rightarrow 0$ as $d\left(p, p_{0}\right) \rightarrow \infty$. Analysing the distance function of $\mathbb{R}^{n}$ restricted to $M$ he concludes that $M$ is properly immersed and that, outside a compact set, $M$ is transversal to the spheres $S_{r}$

Research partially supported by CNPq-Brasil 
of $\mathbb{R}^{n}$, with radius $r$ and centered in $p_{0}$. In particular $M$ is of finite topological type. Also, the flatness of $\mathbb{R}^{n}$ allows him to conclude the conformal type of $M$ is finite. We state the result of [2] which will be our main concern.

Theorem 0.1 (Anderson). Let $M^{m} \hookrightarrow \mathbb{R}^{n}$ be a cmi and suppose that $\int_{M}|\mathcal{A}|^{m}$ $<\infty$. Then $M$ is $C^{\infty}$-diffeomorphic to a closed manifold $\bar{M}$ with a finite numbers of points removed. Also the Gauss map $g: M \rightarrow G_{n, m}$ extends to $a C^{n-2}$ map $\bar{g}: \bar{M} \rightarrow G_{n, m}$ and the metric on $M$ extends conformally to a metric of class $C^{n-2}$ of $\bar{M}$.

Thus each end of $M^{m}$ is diffeomorphic to $S^{m-1} \times[0, \infty)$. Furthermore, Anderson proves also that in the case $m \geq 3$ all ends are embedded.

It is natural to consider the above problem when the ambient space is $\mathbb{H}^{n}$. We make use of the Sobolev inequalities [12] and of Simons equation [17] for the Laplacian of $\mathcal{A}$ on $M$ to show that $|\mathcal{A}|(p)$ goes to zero as $\operatorname{dist}_{M}\left(p, p_{0}\right) \rightarrow \infty$, $p_{0}$ a fixed point of $M$. We do not have an estimate for the decreasing rate of $|\mathcal{A}|$ as good as in the Euclidean case, but the properties of the distance function of $\mathbb{H}^{n}$ restricted to $M$ will allow us to bypass the absence of homotheties in $\mathbb{H}^{n}$ to conclude that $M$ is properly immersed and meets transversally the geodesic spheres $S_{r}$ of $\mathbb{H}^{n}$, at least outside some compact set of $M$.

For the special case of a $\mathrm{cmi} M^{2} \hookrightarrow \mathbb{H}^{n}$, we prove that $M$ cannot have finite conformal type. Also we prove that the index of the operator $\mathbf{L}=-\Delta+2-|\mathcal{A}|^{2}$ is finite. When $n=3$ this is just the stability operator. This extends in one direction a result of Fisher-Colbrie [6], namely, finite total "extrinsic" curvature $\int_{M}|\mathcal{A}|^{2}<\infty$ implies the index of $M$ is finite (the reciprocal assertion fails in the hyperbolic case). Here are the main results we will prove in this paper.

Theorem A. Let $\varphi: M^{m} \hookrightarrow \mathbb{H}^{n}$ be a complete minimal immersion of a connected m-dimensional manifold $M$. Suppose that $\int_{M}|\mathcal{A}|^{m}<\infty$. Then $M$ is properly immersed and is diffeomorphic to the interior of a compact manifold $\bar{M}$ with boundary. Furthermore $\varphi$ extends to a continous map $\bar{\varphi}: \bar{M} \hookrightarrow \overline{\mathbb{H}}^{n}$, $\overline{\mathbb{H}}^{n}$ the compactified of $\mathbb{H}^{n}$. 
In the case of a minimal surface $M$ we have information about the conformal type and the asymptotic behavior of $M$.

Theorem B. Let $M^{2} \hookrightarrow \mathbb{H}^{n}$ be a complete connected minimal surface with $\int_{M}|\mathcal{A}|^{2}<\infty$. Then $M$ is conformally equivalent to a compact surface $\bar{M}$ with a finite number of disks removed and the index of the operator $\mathbf{L}=$ $-\Delta+2-|\mathcal{A}|^{2}$ is finite. Furthermore the asymptotic boundary $\partial_{\infty} M$ is a Lipschitz curve.

We remark that the asymptotic behaviour of an immersion $M^{m_{\hookrightarrow}} \mathbb{H}^{n}$ as above is very different from the situation in $\mathbb{R}^{n}$. In fact, any compact closed submanifold $V^{n-2} \subset \mathbb{H}^{n}$ of class $C^{2+\alpha}, \alpha>0$, can be realized as the asymptotic boundary of a minimizing rectifiable current $T^{n-1}$ of $\mathbb{H}^{n}$ [1]. The regularity result of Hardt-Lin [11] states that such a current is of class $C^{2+\beta}, \beta>0$, in a neighbourhood of the sphere at infinity $\partial_{\infty} \mathbb{H}^{n}$. When $n \leq 7, T^{n-1}$ is a smooth submanifold of $\mathbb{H}^{n}$. A direct calculation shows us that for a $\mathrm{cmi} M^{m} \hookrightarrow \mathbb{H}^{n}$, which extends to a $C^{2}$-submanifold of $\overline{\mathbb{H}}^{n}$, we always have $\int_{M}|\mathcal{A}|^{m}<\infty$. This provides us with a lot of hypersurfaces satisfying the hypotheses of theorem A and having arbitrary topological type at infinity, as long as $n \leq 7$.

In view of theorem $B$ a natural question arises : how regular at infinity is a surface satisfying the hypotheses of theorem B? It seems to the author that a $C^{1}$ regularity up to the boudary is necessary.

In section 1 we establish some notations and we prove a result about the essential spectrum of the Schrödinger operator over a complete Riemannian manifold. The index of this operator is also defined. In section 2 we develop the basic properties of the distance function of $\mathbb{H}^{n}$ when restricted to a submanifold. We prove a compactification theorem for submanifolds whose second fundamental form is small outside some compact set. In section 3 we prove the analytical part of theorem A and B and we make use of the results in section 2 to conclude the topological type is finite and that the immersion extends continuously to the compactified of $M$. The assertion about the conformal type in theorem $\mathrm{B}$ is proved in section 4.

This paper is based upon the author's Doctorat dissertation at Université Paris VII. I would like to express my gratitude to my advisor, H. Rosenberg, 
for useful conversations and valuable advice.

\section{Notation And Known Results}

1.1. Minimal submanifolds. Let $M^{m} \hookrightarrow N^{n}$ be a immersion of a $m$-dimensional manifold $M$ into a $n$-dimensional Riemannian manifold $N$. Consider $M$ with the metric induced by this immersion and denote by $\tilde{\nabla}$ and $\nabla$ the LeviCivita connexions of $N$ and $M$ respectively. For $p \in M$, the tangent space $T_{p} N$ of $N$ at $p$ splits as an orthogonal direct sum $T_{P} N=T_{p} M \oplus \mathcal{N}_{p}(M)$, where $\mathcal{N}_{p}(M)$ is the normal fiber to $M$ at $p$. The second fundamental form of the immersion is the symmetric bilinear form over $T_{p} M$ defined by

$$
\mathcal{A}\left(X_{p}, Y_{p}\right)=\left(\tilde{\nabla}_{X} Y\right)^{\perp}(p) \quad ; \quad X_{p}, Y_{p} \in T_{p} M
$$

where $X, Y$ are extensions of $X_{p}$ and $Y_{p}$ which are tangent to $M$.

Let us consider $\mathcal{A}$ as an element of $\operatorname{Hom}\left(\mathcal{S}_{p}(M), \mathcal{N}_{p}\right)$ where $\mathcal{S}_{p}(M)$ is the space of symmetric linear endomorphisms of $T_{p} M$. For the natural internal product of $\mathcal{S}_{p}(M)$ and $\mathcal{N}_{p}(M)$, let $\mathcal{A}_{p}^{t} \in \operatorname{Hom}\left(\mathcal{N}_{p}, \mathcal{S}_{p}(M)\right)$ be the transpose of $\mathcal{A}$ and set $\mathcal{B}_{p}=\mathcal{A}_{p} \circ \mathcal{A}_{p}^{t}$. The norm of this application is by definition the norm $|\mathcal{A}|$ of $\mathcal{A}$. If $\left\{e_{i}\right\}_{i=1, \ldots, m}$ is an orthonormal frame of $T_{p}(M)$ then

$$
|\mathcal{A}|^{2}(p)=\sum_{i, j=1}^{m}\left|\mathcal{A}\left(e_{i}, e_{j}\right)\right|^{2}
$$

The trace of $\mathcal{A}$ is called the mean curvature $H$ of $M$. With respect to the frame $\left\{e_{i}\right\}_{i=1, \ldots, m}$ we have

$$
H=\frac{1}{m} \sum_{i=1}^{m} \mathcal{A}\left(e_{i}, e_{i}\right)
$$

The immersion $M^{m_{\hookrightarrow}} N^{n}$ is called minimal if $H \equiv 0$. This is equivalent to saying that the immersion is a critical point for the volume functional, i.e., for any compact $K \subset M$ with piecewise smooth boundary, and for any piecewise smooth variation $F: I \times M \rightarrow N$ of $\phi$, which leaves the exterior of $K$ unchanged, we have $\frac{d V}{d t}(0)=0$, where $V(t)$ is the volume of the submanifold $F(t, K)$.

If $M^{m} \hookrightarrow N^{n}$ is minimal, a domain $U \subset M$ is called stable if for any variation $F$ as above whose variation vector field $E=\left.F_{*} \frac{\partial}{\partial t}\right|_{t=0}$ is normal to $M$ and compactly supported in $U$ we have $\frac{d^{2} V}{d t^{2}}(0) \geq 0$. 
Let $\tilde{\mathcal{R}}$ denote the curvature tensor of $N$ and for $v \in \mathcal{N}_{p}(M)$ define $\mathcal{R}(v)$ by $\mathcal{R}(v)=\sum_{i=1}^{m}\left(\widetilde{\mathcal{R}}_{e_{i}, v} e_{i}\right)^{\perp}$. Note that for $v$ unitary $\langle\mathcal{R}(v), v\rangle$ is just the Ricci curvature $\operatorname{Ric}(v)$ of $N$ in the direction of $v$. If $F$ is a normal variation of a minimal surface as above we have [14],

$$
\frac{d^{2} V}{d t^{2}}(0)=-\int_{U}\langle\Delta E+\mathcal{R}(E)+\mathcal{B}(E), E\rangle
$$

In the case of a minimal oriented surface $M^{2} \hookrightarrow H^{3}$ the variation vector field is just $E=\xi \nu$, where $\nu$ is the normal vector of the immersion and $\xi$ is a compactly supported function on $M$. So $M$ is stable if

$$
Q(\xi, \xi)=\int_{M}\left(|\nabla \xi|^{2}+2 \xi^{2}-|\mathcal{A}|^{2} \xi^{2}\right) \geq 0
$$

for all $\xi \in C_{0}^{\infty}(M)$.

1.2. Compactification of Hyperbolic Space. Two oriented rays $\gamma_{1}(s)$ and $\gamma_{2}(s)$ of $\mathbb{H}^{n}$ are said to be equivalent if there exists a real number $c$ such that $d\left(\gamma_{1}(s), \gamma_{2}(s)\right) \leq c$ for all $s \geq 0$, where $d(p, q)$ denotes the hyperbolic distance between the points $p$ and $q$. The sphere at infinity $\partial_{\infty} \mathbb{H}^{n}$ is defined as the space of equivalent classes of oriented rays. For a fixed point $O \in \mathbb{H}^{n}$, identify $\partial_{\infty} \mathbb{H}^{n}$ with the unit sphere $U_{1} \subset T_{O} \mathbb{H}^{n}$ in the following way: for a unit vector $v \in U_{1}$ associate the equivalent class of the ray $\exp _{O} s v, s \geq 0$. This provides $\partial_{\infty} \mathbb{H}^{n}$ with a conformal sructure which is independent of the chosen point $O$. With this structure any isometry of $\mathbb{H}^{n}$ extends conformally to $\overline{\mathbb{H}}^{n}=\mathbb{H}^{n} \cup \partial_{\infty} \mathbb{H}^{n}$.

For $p \in \mathbb{H}^{n} \backslash\{O\}$ we define a "projection" $P: \mathbb{H}^{n} \backslash\{O\} \rightarrow U_{1}$ by $P(p)=$ $\exp _{O}^{-1}(p) /\left|\exp _{O}^{-1}(p)\right|$. Let $S_{r}$ be the geodesic sphere of $\mathbb{H}^{n}$ of radius $r$ and centered at $O$. If $v_{p} \in T_{p}\left(S_{r}\right)$, a comparison between the Jacobi fields along geodesics gives

$$
\left|d \exp _{O}^{-1}\left(v_{p}\right)\right|=\frac{r\left|v_{p}\right|}{\sinh r}
$$

where in the left term the norm is the Euclidean norm of $T_{O} \mathbb{H}^{n}$ and in the right $\left|v_{p}\right|$ is the norm of $v_{p} \in T_{p} \mathbb{H}^{n}$. Thus, for a vector $v_{p} \in T_{p}\left(S_{r}\right)$ we get

$$
\left|(d P)\left(v_{p}\right)\right|=\frac{\left|v_{p}\right|}{\sinh r}
$$


1.3. The spectrum of the Schrödinger operator. Let $M$ be a Riemannian manifold and let $q$ be a real smooth function. The operator $\mathbf{L}=-\Delta+q$ is formally self-adjoint over $C_{c}^{\infty}(M)$, where $\Delta$ is the Laplacian on $M$. When $q$ is bounded below by a real constant and $M=\mathbb{R}^{n}$, Glazman [9] proved that $\mathbf{L}$ admits a unique self-adjoint extension to an unbounded operator on $L^{2}(M)$. The theorem of Dodziuk [5] stated below allows us to follow the steps of the Glazman's proof in the case of an arbitrary manifold $M$. For the sake of completeness we prove this generalization of Glazman's result and we also prove a theorem about the essential spectrum of $\mathbf{L}$.

Theorem 1.1 (Dodziuk). Let $M$ be a complete Riemannian manifold and let $q \in C^{\infty}(M)$ be a real function bounded below by a constant. Suppose $\phi \in C^{\infty}(M) \cap L^{2}(M)$ and $\mathbf{L} \phi \in L^{2}(M)$. Then $\nabla \phi \in L^{2}(M)$ and the functions $\phi \overline{\Delta \phi}, q|\phi|^{2}$ belong to $L^{1}(M)$. Also

$$
(-\Delta \phi, \phi)=(\nabla \phi, \nabla \phi) \quad \text { and } \quad(\mathbf{L} \phi, \phi)=(\nabla \phi, \nabla \phi)+(q \phi, \phi)
$$

where $(.,$.$) is the product of L^{2}(M)$.

Theorem 1.2. Let $M$ be a complete Riemannian manifold and let $q \in C^{\infty}(M)$ be a real function bounded below by a constant. Then the operator $\mathbf{L}=-\Delta+q$ admits a unique self-adjoint extension to an unbounded operator on $L^{2}(M)$.

Proof. It suffices to prove that the spaces $\mathcal{K}_{ \pm}=\operatorname{Image}(\mathbf{L} \pm i I)^{\perp}$ are trivial. Take $\phi \in \mathcal{K}_{+}$. As a distribution, $\phi$ satisfies the equation $\mathbf{L} \phi=i \phi$. For any relatively compact domain $\Omega \subset M$, the operator $\mathbf{L}$ is strictily elliptic. By the Friedrichs's regularity result [7] we have $\phi \in C^{\infty}(M) \cap L^{2}(M)$. Therefore, by the Dodziuk's theorem stated above we obtain

$$
(\mathbf{L} \phi, \phi)=|\phi|^{2}+(q \phi, \phi)=i|\phi|^{2}
$$

But $q$ is a real function, so $\phi \equiv 0$ and $\mathcal{K}_{+}=\{0\}$. Analogously we have $\mathcal{K}_{-}=\{0\}$.

Recall that for a self-adjoint operator $\mathbf{L}$ on a Hilbert space, the essential spectrum ess $(\mathbf{L})$ is the set of points $\lambda \in \mathbb{R}$ such that there exists a bounded 
non-compact sequence $\left\{u_{n}\right\}_{n \in \mathbb{N}}, u_{n} \in$ Domain $(\mathbf{L})$, satisfying

$$
\lim _{n \rightarrow \infty}\left\|(\mathbf{L}-\lambda I) u_{n}\right\|=0 .
$$

A sub-sequence of $\left\{u_{n}\right\}_{n \in \mathbb{N}}$ for which there is no convergent sub-sequence is called characteristic for $(\lambda, \mathbf{L})$.

Now let $\mathbf{L}=-\Delta+q$ be as in theorem 1.2 and let $N$ be a domain of $M$ which is relatively compact and has $C^{\infty}$ boundary. Consider the operator $l_{N}=-\Delta+q$ defined on $C^{\infty}(M \backslash N)$. The quadratic form $Q(\phi)=\left(l_{N} \phi, \phi\right)$ defined on $C_{0}^{\infty}(M \backslash N)$ is bounded below, so it admits a closed extension. We define $\mathbf{L}_{N}$ to be the Friedrichs's extension of $l_{N}$, determined by the closed extension of $Q$. We prove now the generalization of Glazman's theorem [9], p. 68. When $q \equiv 0$ this result was obtained by Donnelly [4].

Theorem 1.3. $\operatorname{ess}(\mathbf{L}) \subset \operatorname{ess}\left(\mathbf{L}_{N}\right)$.

Proof. Suppose the sequence $\left\{u_{n}\right\}_{n \in \mathbb{N}}$ is characteristic for $(\lambda, \mathbf{L})$. Without loss of generality we can suppose it is an orthonormal characteristic sequence for $(\lambda, \mathbf{L})$. By virtue of theorem 1.2 (the operator is essentially self-adjoint) there exists $\left\{\phi_{n}\right\}_{n \in \mathbb{N}}, \phi_{n} \in C_{0}^{\infty}(M)$, such that, for $n \in \mathbb{N}$,

$$
\left\|\phi_{n}-u_{n}\right\| \leq \frac{1}{n} \quad \text { and } \quad\left\|\mathbf{L} \phi_{n}-\mathbf{L} u_{n}\right\| \leq \frac{1}{n}
$$

This implies $\left\{\phi_{n}\right\}_{n \in \mathbb{N}}$ is also characteristic for $(\lambda, \mathbf{L})$ and in particular we have

$$
\left(\mathbf{L} \phi_{n}-\lambda \phi_{n}, \phi_{n}\right) \rightarrow 0 \text { as } n \rightarrow \infty
$$

So for $n$ large enough we get

$$
\left\|\nabla \phi_{n}\right\|^{2}+\left(q \phi_{n}, \phi_{n}\right)-\lambda\left\|\phi_{n}\right\|^{2} \leq 1
$$

Let $-q_{0}$ be a lower bound for $q$. We obtain, for $n$ large,

$$
\left\|\nabla \phi_{n}\right\|^{2} \leq q_{0}\left(\phi_{n}, \phi_{n}\right)+\lambda\left\|\phi_{n}\right\|^{2}+1
$$

Thus $\left\{\phi_{n}\right\}_{n \in \mathbb{N}}$ is bounded in $W^{1,2}(M)$, the space of functions $f$ with $f$ and $\nabla f$ belonging to $L^{2}(M)$. Let $\Omega^{\prime}$ be a relatively compact neighbourhood of $N$. The embedding $W^{1,2}\left(\Omega^{\prime}\right) \hookrightarrow L^{2}\left(\Omega^{\prime}\right)$ is compact, so there exists a sub-sequence $\left\{\phi_{n}^{\prime}\right\}_{n \in \mathbb{N}}$ such that $\phi_{n}^{\prime} / \Omega^{\prime}$ converges in $L^{2}\left(\Omega^{\prime}\right)$. Set $\omega_{n}=\phi_{2 n+1}^{\prime}-\phi_{2 n}^{\prime}$ and remark that $\omega_{n}$ is still characteristic for $(\lambda, \mathbf{L})$ and that $\omega_{n} \rightarrow 0$ in $L^{2}\left(\Omega^{\prime}\right)$. Let 
$\Omega$ be a neighbourhood of $N$ such that $N \subset \bar{\Omega} \subset \Omega^{\prime}$ and let $\xi \in C_{0}^{\infty}\left(\Omega^{\prime}\right)$ be such that $\xi=1$ on $\Omega$. We have

$$
\left(\mathbf{L} \omega_{n}-\lambda \omega_{n}, \xi^{2} \omega_{n}\right) \rightarrow 0 \quad \text { as } n \rightarrow \infty
$$

and by Dodziuk's theorem

$$
\left\|\xi \nabla \omega_{n}\right\|^{2}+\left(\xi \omega_{n}, 2 \omega_{n} \nabla \xi\right)+\left(q \xi \omega_{n}, \xi \omega_{n}\right)-\lambda\left(\xi \omega_{n}, \xi \omega_{n}\right) \rightarrow 0 \quad \text { as } n \rightarrow \infty
$$

Since $\left\|\omega_{n}\right\|_{L^{2}\left(\Omega^{\prime}\right)} \rightarrow 0$ and $\operatorname{support}(\xi) \subset \Omega^{\prime}$ we get $\left\|\nabla \omega_{n}\right\|_{L^{2}(\Omega)} \rightarrow 0$ as $n \rightarrow \infty$.

This allows us to construct a characteristic sequence for $(\lambda, \mathbf{L})$ which vanishes on a neighbourhood of $N$. As a matter of fact, let $U$ be a neighbourhood of $N$ such that $\bar{U} \subset \operatorname{int}(\Omega)$, and let $\theta$ be a smooth function which satisfies $0 \leq \theta \leq 1, \theta=0$ in $U$ and $\theta=1$ in $M \backslash \Omega$. Set $v_{n}=\theta \omega_{n}, n \in \mathbb{N}$, and remark that $v_{n} \in C_{0}^{\infty}(M \backslash N)$. Also the sequence $\left\{v_{n}\right\}_{n \in \mathbb{N}}$ is bounded and non-compact and

$$
\begin{array}{r}
\left\|\mathbf{L} v_{n}-\lambda v_{n}\right\| \leq\left\|\mathbf{L} \omega_{n}-\lambda \omega_{n}\right\|+\left(\sup _{M}|\Delta \theta|\right)\left\|\omega_{n}\right\|_{L^{2}(\Omega)} \\
+2\left(\sup _{M}|\nabla \theta|\right)\left\|\nabla \omega_{n}\right\|_{L^{2}(\Omega)} .
\end{array}
$$

Hence $\left\|\mathbf{L} v_{n}-\lambda v_{n}\right\| \rightarrow 0$ as $n \rightarrow \infty$ and the sequence $\left\{v_{n}\right\}_{n \in \mathbb{N}}$ is characteristic for $\left(\lambda, \mathbf{L}_{N}\right)$.

For a Riemannian manifold $M$ and an operator $\mathbf{L}$ as in theorem 1.3 we define the index of $\mathbf{L}$ in the following way: Let $\Omega$ be a relatively compact domain of $M$ with piecewise smooth boundary. The number of negative engeinvalues for the Dirichlet problem

$$
\mathbf{L} u=\lambda u \quad ; \quad u / \partial \Omega=0
$$

is finite. Let $\operatorname{ind}_{\Omega}(\mathbf{L})$ be this number. Consider an exhaustion $\left\{\Omega_{n}\right\}_{n \in \mathbb{N}}$ of $M$ by relatively compact domains with piecewise smooth boundary. The index $\operatorname{ind}_{M}(\mathbf{L})$ of $\mathbf{L}$ is defined by

$$
\operatorname{ind}_{M}(\mathbf{L})=\lim _{n \rightarrow \infty} \operatorname{ind}_{\Omega_{n}}(\mathbf{L})
$$

This limit does not depend on the chosen exhaustion [6], so the $\operatorname{ind}_{M}(\mathbf{L})$ is well defined. 


\section{Submanifolds of Hyperbolic Space}

In this section we develop some properties of the distance function of $\mathbb{H}^{n}$ restricted to a submanifold. In particular we will prove the following result:

Theorem 2.1. Let $M^{m} \hookrightarrow \mathbb{H}^{n}$ be a complete immersion of a connected manifold $M$. Suppose there exists $\epsilon<1$ and a compact set $C \subset M$ such that $|\mathcal{A}|(p) \leq \epsilon$ for $p \in M \backslash C$. Then the immersion is proper and for $r$ large enough $M$ is transversal to the geodesic spheres $S_{r}$ of $\mathbb{H}^{n}$. In particular $M$ is diffeomorphic to the interior of a compact manifold with boundary $\bar{M}$. Furthermore the immersion $\phi$ extends to a continuous map $\bar{\phi}: \bar{M} \hookrightarrow \overline{\mathbb{H}}^{n}$.

2.1. The distance function of $\mathbb{H}^{n}$ restricted to submanifolds. Let $O \in$ $\mathbb{H}^{n}$ be a fixed point and let $M^{m} \hookrightarrow \mathbb{H}^{n}$ be a isometric immersion. Let $d(q)$ be the distance of $q \in \mathbb{H}^{n}$ to $O$ and let $r$ be the restriction of $d$ to $M$. Denote by $\tilde{\nabla}$ and $\nabla$ the Levi-Civita connexions of $\mathbb{H}^{n}$ an $M$ respectively. Let $\frac{\partial}{\partial d}=\widetilde{\nabla} d$ denote the unitary radial vector field centered at $O$ and defined on $\mathbb{H}^{n} \backslash\{O\}$.

For $p \in M$ let $\left\{E_{i}\right\}_{i=1, \ldots, m}$ be a frame tangent to $M$, defined in a neighbourhood of $p \in M$, orthonormal at $p$ and satisfying $\nabla_{E_{i}} E_{j}(p)=0$, for $i, j=1, \ldots, m$. For $j=1, \ldots, m$ we have $E_{j} r=\left\langle\frac{\partial}{\partial d}, E_{j}\right\rangle$, so

$$
E_{i} E_{j} r=\left\langle\widetilde{\nabla}_{E_{i}} \frac{\partial}{\partial d}, E_{j}\right\rangle+\left\langle\frac{\partial}{\partial d}, \widetilde{\nabla}_{E_{i}} E_{j}\right\rangle \quad ; \quad i, j=1, \ldots, m
$$

where $\langle$,$\rangle is the metric of \mathbb{H}^{n}$. Recall that for a vector $v \in T_{p} S_{r}$ we have, $\widetilde{\nabla}_{v} \frac{\partial}{\partial d}=\operatorname{coth}(r) v$. As $\tilde{\nabla}_{\frac{\partial}{\partial d}} \frac{\partial}{\partial d}=0$, we obtain, for $i=1, \ldots, m$

$$
\left(\widetilde{\nabla}_{E_{i}} \frac{\partial}{\partial d}\right)(p)=\left(E_{i}-\left\langle E_{i}, \frac{\partial}{\partial d}\right\rangle \frac{\partial}{\partial d}\right) \operatorname{coth} r(p)
$$

and for $i, j=1, \ldots, m$ we get at $p$

$$
E_{i} E_{j} r=\left(\delta_{i j}-\left\langle E_{i}, \frac{\partial}{\partial d}\right\rangle\left\langle E_{j}, \frac{\partial}{\partial d}\right\rangle\right) \operatorname{coth} r+\left\langle\frac{\partial}{\partial d}, \widetilde{\nabla}_{E_{i}} E_{j}\right\rangle
$$

If the frame $\left\{E_{i}\right\}_{i=1, \ldots, m}$ is orthonormal, the Laplacian of $r$ at $p$ is given by $\Delta r(p)=\sum_{i=1}^{m}\left(E_{i} E_{j} r\right)(p)$. Hence we obtain, at all $p \in M, p \neq O$,

$$
\Delta r=\left(m-|\nabla r|^{2}\right) \operatorname{coth} r+m\left\langle\frac{\partial}{\partial d}, H\right\rangle
$$


In the special case where $M$ is a curve $\gamma \subset \mathbb{H}^{n}$ parametrized by the arc length $s$ we get

$$
r^{\prime \prime}(s)=\left(1-\left(r^{\prime}(s)\right)^{2}\right) \operatorname{coth} r+\left\langle\frac{\partial}{\partial d}, \tilde{\nabla}_{\gamma^{\prime}(s)} \gamma^{\prime}(s)\right\rangle .
$$

A straightforward consequence of the above equations are the following lemmas:

Lemma 2.2. Let $M^{m} \hookrightarrow \mathbb{H}^{n}$ be an immersion and let $p \in \operatorname{int}(M)$ be a critical point of $r$. Suppose $|\mathcal{A}|(p) \leq 1$. Then $p$ is a point of strict minimum for $r$.

Proof. Let $\left\{e_{i}\right\}_{i=1, \ldots, m}$ be a orthonormal frame of $T_{p} M$ and let $x$ be the normal coordinate system adapted to $\left\{e_{i}\right\}_{i=1, \ldots, m}$, i.e., $x=\chi \circ\left(\exp _{p}\right)^{-1}$, where $\chi: T_{p} M \rightarrow \mathbb{R}^{n}$ is given by $\chi\left(\sum_{i=1}^{m} y^{i} e_{i}\right)=\left(y^{1}, \ldots, y^{m}\right)$. Since $\nabla r(p)=0$ we can choose the frame $\left\{e_{i}\right\}_{i=1, \ldots, m}$ such that $\frac{\partial^{2} r}{\partial x_{i} \partial x_{j}}(p)=0$, for $i \neq j$. Setting $E_{i}=\frac{\partial}{\partial x_{i}}, i=1, \ldots, m$, by equation (2.2) we get

$$
E_{i} E_{i} r=\operatorname{coth} r+\left\langle\frac{\partial}{\partial d}, \tilde{\nabla}_{E_{i}} E_{i}\right\rangle .
$$

As $\nabla_{E_{i}} E_{i}(p)=0$ we have $\left|\widetilde{\nabla}_{E_{i}} E_{i}(p)\right|=\left|\left(\widetilde{\nabla}_{E_{i}} E_{i}(p)\right)^{\perp}\right| \leq|\mathcal{A}|(p) \leq 1$. So $E_{i} E_{i} r(p)>0$, for $i=1, \ldots, m$, which implies, by the Taylor series expansion of $r$, that $p$ is a point of strict minimum.

Lemma 2.3. Let $\gamma:[0, l) \subset \mathbb{H}^{n}, 0<l \leq \infty$, be a curve parametrized by the arc length s. Suppose the geodesic curvature of $\gamma$ satisfies $\left|\widetilde{\nabla}_{\gamma^{\prime}(s)} \gamma^{\prime}(s)\right| \leq \epsilon$, for some $\epsilon<1$. Then $d(\gamma(0), \gamma(s)) \geq \sqrt{1-\epsilon} s, 0 \leq s<l$.

Proof. First observe that the curve $\gamma$ is necessarily embedded ; otherwise, taking the intersection point as the origin of $\mathbb{H}^{n}$, the distance function $r(s)$ defined over $\gamma$ would have a interior maximum, which contradicts lemma 2.2. Now taking the origin to be the point $\gamma(0)$, equation (2.4) says that

$$
r^{\prime \prime}(s)=\left(1-\left(r^{\prime}(s)\right)^{2}\right) \operatorname{coth} r(s)+\left\langle\frac{\partial}{\partial d}, \tilde{\nabla}_{\gamma^{\prime}(s)} \gamma^{\prime}(s)\right\rangle
$$

for all $0<s<l$. Also $r(0)=0$ and $\lim _{s \rightarrow 0} r^{\prime}(s)=1$. It suffices now to prove that $r^{\prime}(s) \geq \sqrt{1-\epsilon}$, for all $0<s<l$. Suppose this is not the case and let $s_{1}$ be the smallest positive real number for which $r^{\prime}\left(s_{1}\right)=\sqrt{1-\epsilon}$. Then

$$
r^{\prime \prime}\left(s_{1}\right)=\epsilon \operatorname{coth} r\left(s_{1}\right)+\left\langle\frac{\partial}{\partial d}, \tilde{\nabla}_{\gamma^{\prime}\left(s_{1}\right)} \gamma^{\prime}(s)\right\rangle
$$


which, under the hypotheses of the lemma, implies $r^{\prime \prime}\left(s_{1}\right)>0$. But this implies the existence of $s_{0}, 0<s_{0}<s_{1}$, with $r^{\prime}\left(s_{0}\right)<r^{\prime}\left(s_{1}\right)$, violating the choice of $s_{1}$.

2.2. Proof of theorem 2.1. First we prove that the immersion is proper and tranversal to the geodesic spheres $S_{r}$, for $r$ large.

\subsection{The immersion is proper.}

Proof. Let $\bar{r}=\sup _{q \in C} r(q)$. For $p \in M \backslash C$ let $\gamma$ be a geodesic of $M$, parametrized by arc length, which realises the distance between $C$ and $p$. Say $\gamma(0) \in \partial C$ and $\gamma(l)=p$, where $l$ is the length of $\gamma$. Of course $\gamma(s) \subset M \backslash C$, for all $s \in(0, l]$. As $\gamma$ is a geodesic of $M$, we have $\left|\widetilde{\nabla}_{\gamma^{\prime}(s)} \gamma^{\prime}(s)\right| \leq|\mathcal{A}|(\gamma(s))$, $s \in[0, l]$. From lemma 2.3 we obtain

$$
\begin{aligned}
r(p)=d(O, p) & \geq d(\gamma(0), \gamma(l))-d(O, \gamma(0)) \\
& \geq \sqrt{1-\epsilon} l-\bar{r} .
\end{aligned}
$$

Thus when $d(p, C)$ goes to infinity we get $r(p) \rightarrow \infty$, which means the immersion is proper.

\section{4. $\mathrm{M}$ is transversal to $\mathrm{S}_{r}$ for $\mathbf{r}$ large.}

Proof. Let $\Omega_{1}=M \cap B_{\bar{r}}$, where $B_{r}$ denotes the closed geodesic ball of $\mathbb{H}^{n}$ of radius $r$. As the immersion is proper, $\Omega_{1}$ is a compact set of $M$ and by definition of $\bar{r},|\mathcal{A}| \leq \epsilon$ in $M \backslash \Omega_{1}$. Suppose that there exists a critical point $p$ of $r$ in $M \backslash \Omega_{1}$. By lemma $2.2 p$ is a strict minimum for $r$. If $\gamma$ is any geodesic joining $p$ to $\partial \Omega_{1}$ then the maximum of the function $r(s)$ over $\gamma(s)$ is greater then $\max (\bar{r}, r(p))$. So this maximum is attained at a point $\bar{p} \in M \backslash \Omega_{1}$, which is impossible by lemma 2.2 applied to the geodesic $\gamma$. This contradiction implies that $r$ has no critical points in $M \backslash \Omega_{1}$, i.e., $M$ is transversal to $S_{r}$ for $r \geq \bar{r}$.

We have therefore a complete proper immersion $M^{m} \hookrightarrow \mathbb{H}^{n}$ such that the function $r$ has no critical points in $M \backslash \Omega_{1}$ where $\Omega_{1}=M \cap B_{\bar{r}}$, for some $\bar{r}>0$. Furthermore $|\mathcal{A}| \leq \epsilon<1$ in $M \backslash \Omega_{1}$. Let $\Sigma(r)=M \cap S_{r}$, so for $r \geq \bar{r}, \Sigma(r)$ is a compact $m-1$ dimensional submanifold of $M$. On $M \backslash \Omega_{1}$ define the fields 
$\xi=\nabla r /|\nabla r|$ and $Y=\nabla r /|\nabla r|^{2}$. Let $\Psi_{t}$ be the flow of $Y$. Thus $\Psi_{t}$ maps $\Sigma(\bar{r})$ diffeomorphically into $\Sigma(\bar{r}+t)$, for $t \geq 0$. For a point $p$ in $\Sigma(\bar{r})$ and $t \geq 0$, define $\alpha(p, t)=\sqrt{1-|\nabla r|^{2}}\left(p_{t}\right)$, where $p_{t}=\Psi_{t}(p)$. For $p \in \Sigma(\bar{r})$ this function satisfies

$$
\frac{1}{2} \frac{\partial}{\partial t} \alpha^{2}=-\left\langle\mathcal{A}(\xi, \xi), \frac{\partial}{\partial d}\right\rangle-\alpha^{2} \operatorname{coth}(\bar{r}+t) .
$$

Moreover if $\eta \in T_{p} \Sigma(r)$, for $r \geq \bar{r}$, we have

$$
\frac{1}{2} \eta\left(\alpha^{2}\right)=-\sqrt{1-\alpha^{2}}\left\langle\mathcal{A}(\eta, \xi), \frac{\partial}{\partial d}\right\rangle .
$$

To see this let $\left\{N_{i}\right\}, i=1, \ldots, k$, be a normal frame to $M$ in a neighbourhood of $p_{t}$, where $k=n-m$ is the codimension of $M$. Write $\nabla r=$ $\frac{\partial}{\partial d}-\sum_{i=1}^{k}\left\langle N_{i}, \frac{\partial}{\partial d}\right\rangle N_{i}$. For a vector $E \in T_{p_{t}} M$ we have

$$
\nabla_{E} \nabla r=\left(\tilde{\nabla}_{E} \frac{\partial}{\partial d}-\sum_{i=1}^{k}\left\langle N_{i}, \frac{\partial}{\partial d}\right\rangle \tilde{\nabla}_{E} N_{i}\right)^{T}
$$

so from equation (2.1) we obtain

$$
\nabla_{E} \nabla r=(E-\langle E, \nabla r\rangle \nabla r) \operatorname{coth} r-\sum_{i=1}^{k}\left\langle N_{i}, \frac{\partial}{\partial d}\right\rangle\left(\widetilde{\nabla}_{E} N_{i}\right)^{T} .
$$

From $\frac{1}{2} \nabla_{E}|\nabla r|^{2}=\left\langle\nabla_{E} \nabla r, \nabla r\right\rangle$ we get

$$
\frac{1}{2} \nabla_{E}|\nabla r|^{2}=\langle E, \nabla r\rangle\left(1-|\nabla r|^{2}\right) \operatorname{coth} r+|\nabla r|\left\langle\frac{\partial}{\partial d}, \mathcal{A}(E, \xi)\right\rangle
$$

where we made use of the fact that $\left\langle\widetilde{\nabla}_{E} N_{i}, \xi\right\rangle=-\left\langle N_{i}, \tilde{\nabla}_{E} \xi\right\rangle$ and that $\nabla r=$ $|\nabla r| \xi$. Taking $E=\xi$ and remarking that $\frac{\partial}{\partial t}=\xi /|\nabla r|$ we obtain

$$
\frac{1}{2} \frac{\partial}{\partial t}|\nabla r|^{2}=\left(1-|\nabla r|^{2}\right) \operatorname{coth} r+\left\langle\frac{\partial}{\partial d}, \mathcal{A}(E, \xi)\right\rangle
$$

which, after replacing $|\nabla r|^{2}$ by $1-\alpha^{2}$, is equation (2.5). In the same way, equation (2.6) is obtained from (2.7) with $E=\eta$.

Now we get the asymptotic behaviour of $|\nabla r|$.

Lemma 2.4. On $M \backslash \Omega_{1}$ the function $|\nabla r|$ satisfies

$$
|\nabla r|^{2}\left(p_{t}\right) \geq(1-\epsilon)\left(1-e^{-2 t}\right) \quad ; \quad \forall p \in \Sigma(\bar{r}) .
$$


Proof. By equation (2.5), for $p \in \Sigma(\bar{r})$ the function $\alpha(t)=\alpha(p, t)$ satisfies, for $t \geq 0$

$$
\frac{1}{2}\left(\alpha^{2}\right)^{\prime}(t) \leq \epsilon-\alpha^{2}(t)
$$

Let $f(t)=\epsilon+(1-\epsilon) \mathrm{e}^{-2 t}$ be the solution of $\frac{1}{2} f^{\prime}(t)+f(t)=\epsilon$, with $f(0)=1$. The function $h(t)=f(t)-\alpha^{2}(t)$ satisfies $\frac{1}{2} h^{\prime}(t)+h(t) \geq 0$ for $t \geq 0$ and $h(0) \geq 0$. This implies that $h(t) \geq 0$, for all $t \geq 0$. Thus for all $p \in \Sigma(\bar{r})$ and $t \geq 0$ we have, at $p_{t}$,

$$
1-|\nabla r|^{2} \leq \epsilon+(1-\epsilon) \mathrm{e}^{-2 t}
$$

We are now able to finish the proof of theorem 2.1.

\subsection{Asymptotic behavior.}

Proof. If $M$ is non orientable we replace $M$ by the orientable double cover of $M$ and remark that the hypotheses $|\mathcal{A}| \leq \epsilon$ outside a compact set is still satisfied. Let $P: \mathbb{H}^{n} \backslash\{O\} \rightarrow U_{1}$ be the projection on the unit sphere of $T_{O} \mathbb{H}^{n}$ as described in section 1 . Denote by $\chi: \Sigma(\bar{r}) \times[0, \infty) \rightarrow U_{1}$ the map $\chi(p, t)=P \circ \Psi_{t}(p)$. We must prove that the 1-parameter family of immersions $\left\{\chi_{t}\right\}$, given by $\chi_{t}(p)=\chi(p, t)$ converges uniformly in $p \in \Sigma(\bar{r})$ as $t \rightarrow \infty$.

Observe that $\left|\frac{\partial \chi}{\partial t}(p, t)\right|=\left|d P\left(\gamma^{\prime}(t)\right)\right|$ where $\gamma(t)=\Psi_{t}(p)$ is the integral curve of $Y$ with $\gamma(0)=p$. From equation (1.2) we have, after projection of the vector $Y$ on $T_{p_{t}} S_{\bar{r}+t}$,

$$
\left|\frac{\partial \chi}{\partial t}(p, t)\right|=\frac{\sqrt{1-|\nabla r|^{2}}}{|\nabla r| \sinh (\bar{r}+t)}
$$

By lemma 2.4, given $\delta$, with $\epsilon<\delta<1$, there exists $t_{1}$ such that for $t \geq t_{1}$, we have $|\nabla r|\left(p_{t}\right) \geq \sqrt{1-\delta}$. From the above equation we get, for $t \geq t_{1}$,

$$
\left|\frac{\partial \chi}{\partial t}(p, t)\right| \leq \frac{1}{\sqrt{1-\delta} \sinh (\bar{r}+t)}
$$

and this inequality implies

$$
\int_{0}^{\infty}\left|\frac{\partial \chi}{\partial t}(p, t)\right| d t \rightarrow 0 \quad \text { as } t \rightarrow \infty
$$

uniformly in $p \in \Sigma(\bar{r})$, so $\chi_{t}$ converges uniformly to a continous map $\chi_{\infty}: \Sigma(\bar{r})$ $\rightarrow \partial_{\infty} \mathbb{H}^{n}$. 
2.6. Immersions transversal to geodesic spheres. Here we consider proper immersions $M^{m_{\hookrightarrow}} \mathbb{H}^{n}$ which are transversal to the geodesic spheres $S_{r}$ of $\mathbb{H}^{n}$ for $r \geq \bar{r}$. We are interested in the volume growth of $\Sigma(r)=M \cap S_{r}$. We suppose $M$ to be orientable and denote by $\omega$ the volume form of $M$. On $M \backslash B(\bar{r})$ define $\sigma=\xi\rfloor \omega$, where $\xi=\nabla r /|\nabla r|$. If $r \geq \bar{r}$ and $\imath_{r}: \Sigma(r) \rightarrow M$ is the inclusion, let $\sigma_{r}=i_{r}^{*} \sigma$ be the volume form of $\Sigma(r)$. Up to sign, $\omega=\xi^{b} \wedge \sigma$, where $\xi^{b}$ is the 1-form dual to the field $\xi$. For $p \in \Sigma(\bar{r})$ and $t \geq 0$ define $f(p, t)$ to be the positive function such that $f(p, t) \sigma_{\bar{r}}=\Psi_{t}^{*} \sigma_{\bar{r}+t}$. By definition of the function $f$ we have, for $s, t \geq 0$ and $p \in \Sigma(\bar{r})$,

$$
\frac{f(p, s+t)}{f(p, t)} \sigma\left(p_{t}\right)=\left(\Psi_{s}^{*} \sigma\right)\left(p_{t}\right) .
$$

Also, as the field $Y=\nabla r /|\nabla r|^{2}$ is invariant by the flow $\Psi_{t}$ we get

$$
\left(\Psi_{t}^{*} \xi^{b}\right)\left(p_{t}\right)=\frac{\left|\nabla r\left(p_{s+t}\right)\right|}{\left|\nabla r\left(p_{t}\right)\right|} \xi^{b}\left(p_{t}\right)
$$

It follows that the Lie derivative of $\omega$ in the direction $Y$ is given by

$$
L_{Y} \omega\left(p_{t}\right)=\frac{1}{f(p, t)}\left(\frac{\partial}{\partial t} f(p, t)-\frac{f(p, t)}{\left|\nabla r\left(p_{t}\right)\right|} \frac{\partial}{\partial t}\left(\left|\nabla r\left(p_{t}\right)\right|\right)\right) .
$$

But $L_{Y} \omega=\operatorname{div} Y \omega$ and $\frac{\partial}{\partial t}\left(\left|\nabla r\left(p_{t}\right)\right|\right)=\frac{1}{\left|\nabla r\left(p_{t}\right)\right|} \xi(|\nabla r|)\left(p_{t}\right)$. Also

$$
\operatorname{div} Y=\frac{1}{|\nabla r|^{2}} \Delta r-\frac{2}{|\nabla r|^{2}} \xi(|\nabla r|)
$$

because $\langle\nabla(|\nabla r|), \nabla r\rangle=|\nabla r| \xi(|\nabla r|)$. From these equations it follows that

$$
f \Delta r=|\nabla r|^{2} \frac{\partial}{\partial t} f+f \xi(|\nabla r|) .
$$

Let $\gamma(s)$ be a integral curve of $\xi$ such that $\gamma(0)=p_{t}$. Let $r(s)=d(\gamma(s))$ and recall that, since $\xi$ is unitary $\left\langle\frac{\partial}{\partial d}, \widetilde{\nabla}_{\gamma^{\prime}(0)} \gamma^{\prime}(s)\right\rangle=\left\langle\frac{\partial}{\partial d}, \mathcal{A}(\xi, \xi)\right\rangle$. From equation (2.4) we have

$$
r^{\prime \prime}(s)=\left(1-\left(r^{\prime}(s)\right)^{2}\right) \operatorname{coth} r(s)+\left\langle\frac{\partial}{\partial d}, \mathcal{A}(\xi, \xi)\right\rangle
$$

and using the fact that $r^{\prime}(s)=|\nabla r|$ we get

$$
\xi(|\nabla r|)=\left(1-|\nabla r|^{2}\right) \operatorname{coth} r+\left\langle\frac{\partial}{\partial d}, \mathcal{A}(\xi, \xi)\right\rangle .
$$


From equations (2.3) and (2.8) we obtain, with $\alpha^{2}=1-|\nabla r|^{2}$, the desired equation for $f$

$$
\frac{1}{f}\left(1-\alpha^{2}\right) \frac{\partial}{\partial t} f=(m-1) \operatorname{coth} r-\left\langle\frac{\partial}{\partial d}, \mathcal{A}(\xi, \xi)\right\rangle+m\left\langle H, \frac{\partial}{\partial d}\right\rangle
$$

\section{Minimal IMMERSiONS}

In this section we prove theorem $\mathrm{A}$ and $\mathrm{B}$. The statement about the conformal type of $M$ in theorem B will be proved in the next section. First we state some basic inequalities.

3.1. Simons and Sobolev inequalities. Let $\varphi: M^{m} \hookrightarrow \mathbb{H}^{n}$ be a $\mathrm{cmi}$ and denote $u=|\mathcal{A}|$. Simons'equation [17], applied to minimal submanifolds of $\mathbb{H}^{n}$, tell us that $u$ satisfies

$$
\Delta u+m u+m u^{3} \geq 0
$$

in the distribution sense. Let $\xi$ be a compactly supported smooth function on $M$ and let $q \geq 1$ be a real number. Multiplying (3.1) by $\xi^{2} u^{2 q-1}$, integrating by parts, rearranging terms and taking square roots we obtain

$$
\left\|\nabla \xi u^{q}\right\|_{2} \leq c_{1} \sqrt{q}\left(\left\|\xi u^{q}\right\|_{2}+\left\|\xi u^{q+1}\right\|_{2}+\left\|u^{q} \nabla \xi\right\|_{2}\right)
$$

for a constant $c_{1}$ which depends only on $m$.

From Sobolev inequality [12], for any smooth function $h$ compactly supported in $M$ we have

$$
\|h\|_{\frac{m}{m-1}} \leq c_{2}\|\nabla h\|_{1}
$$

where $c_{2}$ does not depends on $h$. From (3.3) and the Holder inequality we have, for $1 \leq r<m$,

$$
\|h\|_{\frac{m r}{m-r}} \leq c_{2} \frac{r(m-1)}{m-r}\|\nabla h\|_{r}
$$

These inequalities are valid in case $h$ is a bounded compactly supported function and $h \in W^{1, r}$, the space of functions in $L^{r}(M)$, whose gradient $\nabla h$ also belongs to $L^{r}(M)$. We remark that this is the case when $h=\xi u^{q}, \xi$ a smooth function compactly supported on $M$. 
3.2. Proof of theorem A. We first prove an analytical lemma.

Lemma 3.1. Given $m \geq 2$, there exists universal constants $\epsilon>0$ and $c>0$, depending only on $m$, with the folowing property : If $\varphi: M^{m} \hookrightarrow \mathbb{H}^{n}$ is a minimal immersion of an open manifold $M$, and $x_{0} \in M$ is such that the closed geodesic ball $B(1)$ of radius 1 centered at $x_{0}$ is compact in $M$, then $\left(\int_{B(1)}|\mathcal{A}|^{m}\right)^{\frac{1}{m}} \leq \epsilon$ implies

$$
|\mathcal{A}|\left(x_{0}\right) \leq c\left(\int_{B(1)}|\mathcal{A}|^{m}\right)^{\frac{1}{m}}
$$

Proof. We deal separetely the cases $m \geq 3$ and $m=2$.

case $m \geq 3$. For $\xi \in C_{c}^{\infty}(B(1))$ denote by $\chi$ the characteristic function of the support of $\xi$. If $s>2$ the Holder's inequality gives us

$$
\int_{M} \xi^{2}|\mathcal{A}|^{2} u^{2 q} \leq\left\|\chi|\mathcal{A}|^{2}\right\|_{\frac{s}{2}}\left\|\xi^{2} u^{2 q}\right\|_{\frac{s}{s-2}}=\left\|\chi|\mathcal{A}|^{2}\right\|_{\frac{s}{2}}\left\|\xi u^{q}\right\|_{\frac{2 s}{s-2}}^{2} .
$$

We take $r=2$ in the Sobolev inequality (3.4), apply (3.2) and the above inequality to obtain

$$
\left\|\xi u^{q}\right\|_{\frac{2 m}{m-2}} \leq c_{4} \sqrt{q}\left(\left\|u^{q}|\nabla \xi|\right\|_{2}+\left\|\xi u^{q}\right\|_{2}+\left\|\chi|\mathcal{A}|^{2}\right\|_{\frac{s}{2}}^{\frac{1}{2}}\left\|\xi u^{q}\right\|_{\frac{2 s}{s-2}}\right)
$$

where $c_{4}$ depends only on $m$. Suppose that

$$
c_{4} \sqrt{\frac{m}{2}}\left(\int_{B(1)}|\mathcal{A}|^{m}\right)^{\frac{1}{m}} \leq \frac{1}{2} .
$$

With this assumption, from (3.5) with $s=m$ and $q=\frac{m}{2}$ we have

$$
\left\|\xi u^{\frac{m}{2}}\right\|_{\frac{2 m}{m-2}} \leq c_{5}\left[\left\|u^{\frac{m}{2}} \mid \nabla \xi\right\|_{2}+\left\|\xi u^{\frac{m}{2}}\right\|_{2}\right]
$$

and

$$
\left\|\xi u^{\frac{m}{2}}\right\|_{\frac{2 m}{m-2}} \leq c_{5}\left(\sup _{B(1)}|\nabla \xi|+\sup _{B(1)}|\xi|\right)\left(\int_{B(1)}|\mathcal{A}|^{m}\right)^{\frac{1}{2}}
$$

where $c_{5}=c_{4} \sqrt{2 m}$. Taking $\xi$ such that $0 \leq \xi \leq 1, \xi=1$ on $B\left(\frac{3}{4}\right), \xi=0$ on the exterior of $B(1)$ and such that $|\nabla \xi| \leq 8$, we have by the above inequality

$$
\left\||\mathcal{A}|^{\frac{m}{2}}\right\|_{\frac{2 m}{m-2}, B\left(\frac{3}{4}\right)} \leq 10 c_{5}\left(\int_{B(1)}|\mathcal{A}|^{m}\right)^{\frac{1}{2}}
$$


where the norm in the left side is taken over the ball $B\left(\frac{3}{4}\right)$. We want to use (3.7) to get control of the $L_{\frac{2 m}{m-2}}$ norm of $\xi u^{q}$ in terms of its $L_{2}$ norm.

Let $\epsilon$ be the greatest positive real number such that if $\int_{B(1)}|\mathcal{A}|^{m} \leq \epsilon^{m}$ then inequality (3.6) and

$$
10 c_{5}\left(\int_{B(1)}|\mathcal{A}|^{m}\right)^{\frac{1}{2}} \leq 1
$$

are satisfied. The constant $\epsilon$ depends only on $m$. Remark that for $s=\frac{m^{2}}{m-2}$ we have

$$
\left\||\mathcal{A}|^{2}\right\|_{\frac{s}{2}, B\left(\frac{3}{4}\right)}=\left\||\mathcal{A}|^{\frac{m}{2}}\right\|_{\frac{2 m}{m-2}, B\left(\frac{3}{4}\right)}^{\frac{2}{m}} .
$$

Therefore, assuming $\int_{B(1)}|\mathcal{A}|^{m} \leq \epsilon^{m}$, from (3.5) and (3.7) we get, for $s=\frac{m^{2}}{m-2}$,

$$
\left\|\xi u^{q}\right\|_{\frac{2 m}{m-2}} \leq 2 c_{4} \sqrt{q}\left(\left\|u^{q}|\nabla \xi|\right\|_{2}+\left\|\xi u^{q}\right\|_{2}+\left\|\xi u^{q}\right\|_{\frac{2 s}{s-2}}\right)
$$

for all smooth $\xi$ with support in the ball $B\left(\frac{3}{4}\right)$.

On the other hand, for $s=\frac{m^{2}}{m-2}$, and any $\delta>0$ we have the interpolation formula

$$
\left\|\xi u^{q}\right\|_{\frac{2 s}{s-2}} \leq \delta\left\|\xi u^{q}\right\|_{\frac{2 m}{m-2}}+\delta^{-\sigma}\left\|\xi u^{q}\right\|_{2} .
$$

where $\sigma=\frac{m}{m-2}$. Given $q \geq 1$ we chose $\delta$ such that $c_{4} \delta \sqrt{q}=\frac{1}{4}$. Thus $\delta^{-\sigma}=$ $\left(4 c_{4}\right)^{\sigma} q^{\frac{\sigma}{2}}$ and from (3.9) and (3.10) we get, for any $\xi \in C_{c}^{\infty}\left(B\left(\frac{3}{4}\right)\right)$

$$
\left\|\xi u^{q}\right\|_{\frac{2 m}{m-2}} \leq c_{6} \sqrt{q}\left(\left\|u^{q}|\nabla \xi|\right\|_{2}+\left(1+q^{\frac{\sigma}{2}}\right)\left\|\xi u^{q}\right\|_{2}\right)
$$

for some constant $c_{6}$ which depends only on $m$.

Now we iterate to obtain a bound for $|\mathcal{A}|$ over $B\left(\frac{1}{4}\right)$. For $i \in \mathbb{N}$, let $B_{i}=$ $B\left(\frac{1}{4}+\frac{1}{2^{i+1}}\right)$. Let $\xi_{i}, 0 \leq \xi_{i} \leq 1$, be a Lipschitz function which satisfies

$$
\xi_{i}=1 \quad \text { on } B_{i+1} \quad ; \quad \xi_{i}=0 \quad \text { on } M \backslash B_{i}
$$

and such that $\left|\nabla \xi_{i}\right| \leq 2^{i+2}$. From (3.11) with $\xi=\xi_{i}$ we get

$$
\left\|\xi_{i} u^{q}\right\|_{2 \sigma} \leq c_{6} \sqrt{q}\left(2^{i+3}+q^{\frac{\sigma}{2}}\right)\left\|\chi_{i} u^{q}\right\|_{2}
$$

where $\chi_{i}$ is the characteristic function of $\operatorname{support}\left(\xi_{i}\right)$. Squaring the above inequality we obtain

$$
\left(\int_{B_{i+1}}|\mathcal{A}|^{2 q \sigma}\right)^{\frac{1}{\sigma}} \leq c_{6}^{2} q\left(2^{i+3}+q^{\frac{\sigma}{2}}\right)^{2} \int_{B_{i}}|\mathcal{A}|^{2 q} .
$$


Let $2 q=m \sigma^{i}$ and observe that, for this choice of $q$ we have $c_{6}^{2} q\left(2^{i+3}+q^{\frac{i \sigma}{2}}\right) \leq$ $c_{7}^{i}$ for some constant $c_{7}$ depending only on $m$. Hence from (3.12) we obtain

$$
\left(\int_{B_{i+1}}|\mathcal{A}|^{m \sigma^{i+1}}\right)^{\frac{1}{\sigma}} \leq c_{7}^{i} \int_{B_{i}}|\mathcal{A}|^{m \sigma^{i}}
$$

Define $I_{i}=\left(\int_{B_{i}}|\mathcal{A}|^{m \sigma^{i}}\right)^{\frac{1}{\sigma^{i}}}$. From (3.13) we get $I_{i+1} \leq c_{7}^{\frac{i}{\alpha^{2}}} I_{i}$. Since $\sigma>1$ the series $\sum_{i=1}^{\infty} \frac{i}{\sigma^{i}}$ converges. Thus there exist a real number $c$ depending only $m$ such that

$$
I_{i+1} \leq c^{m} I_{0} .
$$

This implies the norm $L^{\infty}$ of $|\mathcal{A}|^{m}$ over the ball $B\left(\frac{1}{4}\right)$ is bounded by $c^{m} \int_{B(1)}|\mathcal{A}|^{m}$ which is the conclusion of the lemma for $m \geq 3$.

case $m=2$. We prove first there exist $\delta$ such that if $\left(\int_{B(1)}|\mathcal{A}|^{2}\right)^{\frac{1}{2}} \leq \delta$ then the operator $\mathbf{L}=-\Delta+2-|\mathcal{A}|^{2}$ is positive defined on the ball $B\left(\frac{1}{2}\right)$. In the case $n=3$ this means that the ball $B\left(\frac{1}{2}\right)$ is stable.

Let $\xi$ be a smooth compact supported function on $B(1)$ and $\chi$ the characteristic function of the support of $\xi$. From the Sobolev inequality (3.3) we have

$$
\left\|\xi u^{2}\right\|_{2} \leq 2 c_{2}\left(\|\xi u \nabla u\|_{1}+\left\|u^{2} \nabla \xi\right\|_{1}\right)
$$

and by the Cauchy-Schwartz inequality we get

$$
\left\|\xi u^{2}\right\|_{2} \leq 2 c_{2}\|\chi u\|_{2}\left(\|\xi \nabla u\|_{2}+\|u \nabla \xi\|_{2}\right)
$$

Taking $q=1$ in (3.2) and rearranging terms we obtain

$$
\|\xi \nabla u\|_{2} \leq 2 c_{1}\left(\|\xi u\|_{2}+\left\|\xi u^{2}\right\|_{2}+\|u \nabla \xi\|_{2}\right)
$$

and from these last two inequalities we get

$$
\|\xi \nabla u\|_{2} \leq c_{8}\left(\|\xi u\|_{2}+\|\chi u\|_{2}\left(\|\xi \nabla u\|_{2}+\|u \nabla \xi\|_{2}\right)+\|u \nabla \xi\|_{2}\right)
$$

for some constant $c_{8}$ which does not depends on $\xi$. Let $\delta_{1}=\min \left(\frac{1}{2 c_{8}}, 1\right)$ and suppose

$$
\left(\int_{B(1)}|\mathcal{A}|^{2}\right)^{\frac{1}{2}} \leq \delta_{1} .
$$


From (3.15) we get

$$
\|\xi \nabla u\|_{2} \leq 2 c_{8}\left(\|\xi u\|_{2}+2\|u \nabla \xi\|_{2}\right)
$$

for all $\xi$ compactly supported in $B(1)$.

Now take $\xi$ with compact support in $B(1)$ and satisfying

$$
\begin{gathered}
\xi=\left\{\begin{array}{ccc}
1 \quad & ; & \text { on } B\left(\frac{3}{4}\right) \\
0 & ; & \text { on } B(1) \backslash B\left(\frac{3}{4}\right)
\end{array}\right. \\
0 \leq \xi \leq 1, \quad|\nabla \xi| \leq 8 .
\end{gathered}
$$

For any such $\xi$ we have, from (3.17)

$$
\|\xi \nabla u\|_{2} \leq 20 c_{8}\|\chi u\|_{2}
$$

Let $\phi$ be a smooth compactly supported function on $B\left(\frac{1}{2}\right)$ and let $\xi$ be as above, so that (3.18) is verified. From Sobolev inequality (3.3) with $\xi$ replaced by $\xi \phi$ and from Schwartz inequality we have

$$
\frac{1}{2 c_{2}}\|\mathcal{A} \phi \xi\|_{2} \leq\|\xi \nabla|\mathcal{A}|\|_{2}\|\phi\|_{2}+\|\xi \mathcal{A}\|_{2}\|\nabla \phi\|_{2}+\|\phi\|_{2}\|\mathcal{A}|\nabla \xi|\|_{2}
$$

By (3.18) there exist $\delta<\delta_{1}$ such that if

$$
\left(\int_{B(1)}|\mathcal{A}|^{2}\right)^{\frac{1}{2}} \leq \delta
$$

then

$$
\|\xi \nabla|\mathcal{A}|\|_{2}<\frac{1}{4 c_{2}} \quad ; \quad\|\xi \mathcal{A}\|_{2}<\frac{1}{4 c_{2}} \quad ; \quad\|\mathcal{A} \mid \nabla \xi\|_{2}<\frac{1}{4 c_{2}}
$$

Therefore, if $\left(\int_{B(1)}|\mathcal{A}|^{2}\right)^{\frac{1}{2}} \leq \delta$ we have, from (3.19),

$$
\int_{M}|\mathcal{A}|^{2} \phi^{2} \leq 2 \int_{M} \phi^{2}+\int_{M}|\nabla \phi|^{2}
$$

for all $\phi \in C_{c}^{\infty}\left(B\left(\frac{1}{2}\right)\right)$.

Since the immersion is minimal we have by the Gauss equation $K=-1-$ $\frac{1}{2}|\mathcal{A}|^{2}$. Also our surface satisfies the "stability" equation (3.20) on the compact ball of radius $\frac{1}{2}$, the Sobolev inequality (3.3) and Simon's inequality (3.1). So 
we have all the requirements to apply Schoen's stability result [16]: there exists constants $c_{9}, c_{10}$ and $0<\mu \leq \frac{1}{2}$, not depending on the immersion such that

$$
\begin{cases}\int_{B(\mu)}\left(1+|\mathcal{A}|^{2}\right) & \leq c_{9} \\ \sup _{B(\mu)}|\mathcal{A}| & \leq c_{10}\end{cases}
$$

This enable us to find a bound for $|\mathcal{A}|$ on $B\left(\frac{\mu}{4}\right)$ in terms of the $L_{2}$ norm of $|\mathcal{A}|$ on $B(\mu)$. From (3.4) we have, with $r=\frac{4}{3}$,

$$
\left\|\xi|\mathcal{A}|^{q}\right\|_{4} \leq c_{11}\left\|\nabla \xi|\mathcal{A}|^{q}\right\|_{\frac{4}{3}}
$$

for some constant $c_{11}$ and for any function $\xi$ compactly supported in $B(\mu)$. From Holder inequality and the estimate on the area given by (3.21) we get

$$
\left\|\xi|\mathcal{A}|^{q}\right\|_{4} \leq c_{12}\left\|\nabla \xi|\mathcal{A}|^{q}\right\|_{2}
$$

for some constant $c_{12}$ which does not depends on the immersion. By (3.2) and the bound of $|\mathcal{A}|$ on $B(\mu)$ we obtain, for some constant $c_{13}$,

$$
\left\|\xi|\mathcal{A}|^{q}\right\|_{4} \leq c_{13} \sqrt{q}\left(\sup _{B(\mu)}|\xi|+\sup _{B(\mu)}|\nabla \xi|\right)\left\|\chi|\mathcal{A}|^{q}\right\|_{2} .
$$

where $\chi$ is the characteristic function of $B(\mu)$.

An iteration method analogous to that used in the proof of case $m \geq 3$ gives that there exists constants $c_{14}$ and $\epsilon<\delta$ such that if $\left(\int_{B(1)}|\mathcal{A}|^{2}\right)^{\frac{1}{2}} \leq \epsilon$ then

$$
\sup _{B\left(\frac{\mu}{4}\right)}|\mathcal{A}| \leq c_{14}\left(\int_{B(\epsilon)}|\mathcal{A}|^{2}\right)^{\frac{1}{2}}
$$

and this finishes the proof of the lemma.

Now theorem $\mathrm{A}$ is an easy consequence of lemma 3.1. In fact, for a $\mathrm{cmi}$ $M^{m} \hookrightarrow \mathbb{H}^{n}$ satisfying $\int_{M}|\mathcal{A}|^{m}<\infty$, there exists $r_{0}>0$ such that

$$
\left(\int_{M \backslash B\left(r_{0}\right)}|\mathcal{A}|^{m}\right)^{\frac{1}{m}} \leq \epsilon
$$

$\epsilon$ as in lemma 3.1. Thus we have

$$
\sup _{M \backslash B(r+1)}|\mathcal{A}| \leq c\left(\int_{M \backslash B(r)}|\mathcal{A}|^{m}\right)^{\frac{1}{m}}
$$

for $r \geq r_{0}$. Therefore $|\mathcal{A}|(p)$ goes uniformly to 0 as $p \rightarrow \infty$. The result now follows from theorem 2.1 of section 2 . 
Another consequence of lemma 3.1 is a "topological gap phenomenom"

Corollary 3.2. Let $M^{m} \hookrightarrow \mathbb{H}^{n}$ be a connected complete minimal immersion. Then there exists $\bar{\epsilon}$ such that if $\int_{M}|\mathcal{A}|^{m} \leq \bar{\epsilon}$ then $M$ is simply connected.

Proof. It suffices to take $\bar{\epsilon}=\epsilon / c$ for $\epsilon$ and $c$ as in lemma 3.1. Thus $|\mathcal{A}| \leq 1$ on $M$. If $\pi_{1}(M)$ is non trivial then there exists a geodesic $\gamma$ of $M$ with coincidents ending points. As $M$ is minimal $\left|\widetilde{\nabla}_{\gamma^{\prime}} \gamma^{\prime}\right| \leq 1$. The contradiction follows from lemma 2.3. Thus $\pi_{1}(M)$ is trivial.

3.3. Proof of theorem B. The assertion about the conformal type will be proved in the next section.

\section{4. $\partial_{\infty}(M)$ is a Lipschitz.}

Proof. We assume $M$ is orientable. From theorem A we know that a cmi $\varphi: M^{2} \hookrightarrow \mathbb{H}^{n}$ satisfying $\int_{M}|\mathcal{A}|^{2}<\infty$ is properlly immersed and $|\mathcal{A}|(p) \rightarrow 0$ as $p \rightarrow \infty$. In particular $M$ meets transversally the geodesic spheres $S_{r}$ centered at a fixed point $O \in \mathbb{H}^{n}$, for $r>\bar{r}, \bar{r}$ large enough. Let $\Sigma(r)=M \cap S_{r}$ as in section 2. Recall that for $p \in \Sigma(\bar{r})$ and $t \geq 0, f(p, t)$ is the norm of $d \Psi_{t}(\eta(p))$ where $\Psi_{t}$ is the flow of $Y=\nabla r /|\nabla r|^{2}$ and $\eta$ is the unitary vector field defined on $M \backslash B(\bar{r})$ and orthogonal to $\xi=\nabla r /|\nabla r|$. Let $l$ be the length of $\Sigma(\bar{r})$ and let $\gamma:[0, l] \rightarrow \Sigma(\bar{r})$ be a parametrization of $\Sigma(\bar{r})$ by arc length. Define

$$
\begin{gathered}
x:[0, l) \times[0, \infty) \mapsto M \backslash B(\bar{r}) \\
x(\theta, t)=\Psi_{t}(\gamma(\theta))
\end{gathered}
$$

and remark that $\frac{\partial x}{\partial \theta}(\theta, t)=f(\theta, t) \eta(\theta, t)$ where $f(\theta, t)=f(\gamma(\theta), t)$ and $\eta(\theta, t)=$ $\eta(x(\theta, t))$. Also $\frac{\partial x}{\partial t}(\theta, t)=Y(x(\theta, t))$. In the coordinate system given by $x$ the area element is $d S=(f /|\nabla r|) d \theta d t$. Set $\alpha(\theta, t)=\alpha(x(\theta, t))$, where $\alpha=\sqrt{1-|\nabla r|^{2}}$. When $H=0$, equations (2.5), (2.6) and (2.9) give

$$
\begin{gathered}
\frac{1}{2} \frac{\partial}{\partial t} \alpha^{2}=-\left\langle\mathcal{A}(\xi, \xi), \frac{\partial}{\partial d}\right\rangle-\alpha^{2} \operatorname{coth}(\bar{r}+t) \\
\frac{1}{2} \frac{\partial \alpha^{2}}{\partial \theta}=-f\left\langle\mathcal{A}(\eta, \xi), \frac{\partial}{\partial d}\right\rangle \sqrt{1-\alpha^{2}} \\
\frac{1}{f}\left(1-\alpha^{2}\right) \frac{\partial}{\partial t} f=\operatorname{coth}(\bar{r}+t)-\left\langle\frac{\partial}{\partial d}, \mathcal{A}(\xi, \xi)\right\rangle .
\end{gathered}
$$


From (3.22) and (3.24) we obtain

$$
\frac{1}{f} \frac{\partial}{\partial t} f=\operatorname{coth}(\bar{r}+t)+\frac{2 \alpha^{2}}{1-\alpha^{2}} \operatorname{coth}(\bar{r}+t)+\frac{1}{2} \frac{\partial}{\partial t} \ln \left(1-\alpha^{2}\right) .
$$

Assume for the moment that there exists a positive real number $C$ such that

$$
\int_{0}^{\infty} \alpha^{2}(\theta, t) d t \leq C \quad ; \quad \forall \theta \in[0, l] .
$$

Since $|\mathcal{A}|(p) \rightarrow 0$ as $p \rightarrow \infty$ we take $\bar{r}$ large enough to have $|\mathcal{A}| \leq \frac{1}{2}$ and $\alpha^{2} \leq \frac{1}{4}$ on $M \backslash B(\bar{r})$. This is possible by lemma 2.4. Integrating both sides of (3.25) and using (3.26) we obtain

$$
f(p, t)=\mathrm{e}^{t+h(p, t)}
$$

for some bounded function $h(p, t)$ defined on $M \backslash B(\bar{r})$.

Let $\chi: \Sigma(\bar{r}) \times[0, \infty) \rightarrow U_{1}$ be as in the proof of theorem 2.1, $U_{1}$ the unit sphere of $T_{O} \mathbb{H}^{n}$, so that $\chi_{t}(p)=\chi(p, t)$ is just the projection of the curve $\Sigma(\bar{r}+t)$ in the sphere at infinity $\partial_{\infty} \mathbb{H}^{n} \cong U_{1}$. From equation (1.2) we have that

$$
\left|\left(d \chi_{t}\right)(\eta(p))\right|=\frac{f(p, t)}{\sinh r}
$$

and hence, by (3.27), we get a bound for the length of the immersions $\chi_{t}(\Sigma(\bar{r}))$. But a sequence of uniformly convergent curves of $U_{1}$ whose lengths are uniformly bounded converges to a Lipschitz curve of $U_{1}$.

To prove (3.26) we first prove that $\alpha \in L_{2}(M)$.

As $\alpha^{2} \leq \frac{1}{4}$ on $M \backslash B(\bar{r})$ we have $|\nabla r| \geq \frac{3}{4}$ and therefore $f d \theta d t \leq d A \leq$ $\frac{4}{3} f d \theta d t$. Let $D_{t}=\left\{\Psi_{s}(p) \mid p \in \Sigma(\bar{r}) ; 0 \leq s \leq t\right\}$ be the annuli of $M$ bounded by $\Sigma(\bar{r}+t)$ and $\Sigma(\bar{r})$. Since $\left\langle\mathcal{A}(\xi, \xi), \frac{\partial}{\partial d}\right\rangle=\left\langle\left(\widetilde{\nabla}_{\xi} \xi\right)^{\perp},\left(\frac{\partial}{\partial d}\right)^{\perp}\right\rangle$, by the CauchySchwartz inequality we have

$$
\left|\left\langle\mathcal{A}(\xi, \xi), \frac{\partial}{\partial d}\right\rangle\right| \leq|\mathcal{A}| \sqrt{1-|\nabla r|^{2}}=|\mathcal{A}| \alpha
$$

From (3.22) and (3.24) we get

$$
\frac{\partial}{\partial t}\left(\alpha^{2} f\right)+\frac{1-2 \alpha^{2}}{1-\alpha^{2}} f \alpha^{2} \operatorname{coth}(\bar{r}+t)=-\left(\frac{3-\alpha^{2}}{1-\alpha^{2}}\right) f\left\langle\mathcal{A}(\xi, \xi), \frac{\partial}{\partial d}\right\rangle
$$


and, making use of (3.28), we have

$$
\frac{\partial}{\partial t}\left(\alpha^{2} f\right)+\frac{1}{2} \frac{f \alpha^{2}}{|\nabla r|} \leq \frac{4}{3} \frac{f|\mathcal{A}| \alpha}{|\nabla r|} .
$$

Integrating this inequality over $[0, l] \times[0, t]$, using Holder inequality in the right term and remembering that $f(\theta, 0)=1, \forall \theta \in[0, l]$, we obtain

$$
\int_{0}^{l} \alpha^{2} f(\theta, t) d \theta-\int_{0}^{l} \alpha^{2}(\theta, 0) d \theta+\frac{1}{2} \int_{D_{t}} \alpha^{2} \leq \frac{4}{3}\left(\int_{D_{t}}|\mathcal{A}|^{2}\right)^{\frac{1}{2}}\left(\int_{D_{t}} \alpha^{2}\right)^{\frac{1}{2}} .
$$

As $f>0$ this implies that $\alpha \in L_{2}(M)$. By equation (3.24) and the fact that $|\mathcal{A}|<1$ on $M \backslash B(\bar{r})$ we have $\frac{\partial f}{\partial t}>0$ for $t \geq 0$. Thus $f \geq 1$ on $M \backslash B(\bar{r})$. As $\alpha \in L_{2}(M)$, this implies that the integral $\int_{0}^{l} \int_{0}^{\infty} \alpha^{2} d \theta d t$ is finite. Hence for almost all $\theta \in[0, l]$ the integral $\int_{0}^{\infty} \alpha^{2}(\theta, t) d t$ is finite. Changing the parametrization of $\Sigma(\bar{r})$ if necessary we can assume that

$$
\int_{0}^{\infty} \alpha^{2}(0, t) d t<\infty
$$

Define $I(\theta, t)=\int_{0}^{t} \alpha^{2}(\theta, s) d s$. From (3.23) we have for $\theta_{0} \in[0, l]$,

$$
I\left(\theta_{0}, t\right)-I(0, t)=\int_{0}^{\theta_{0}} \frac{\partial I}{\partial \theta}(\theta, t) d \theta \leq 2 \int_{0}^{\theta_{0}} \int_{0}^{t} f|\mathcal{A}| \alpha d t d \theta .
$$

Thus, since $d A \geq f d \theta d t$, we have by the Cauchy-Schwartz inequality

$$
I\left(\theta_{0}, t\right) \leq I(0, t)+2\left(\int_{M}|\mathcal{A}|^{2}\right)^{\frac{1}{2}}\left(\int_{M} \alpha^{2}\right)^{\frac{1}{2}}
$$

\subsection{The operator $\mathbf{L}=-\Delta+2-|\mathcal{A}|^{2}$ has finite index.}

Proof. From theorem A we know that $|\mathcal{A}|(p) \rightarrow 0$ as $p \rightarrow \infty$, so for any $\epsilon \in(0,2)$ there exists a compact set $N_{\epsilon} \subset M$ such that

$$
(\mathbf{L} \phi, \phi) \geq(-\Delta \phi+\epsilon \phi, \phi) \quad \phi \in C_{c}^{\infty}\left(M \backslash N_{\epsilon}\right) \text {. }
$$

Therefore the spectrum of the restriction $\mathbf{L}_{N}$ of $\mathbf{L}$ to the exterior of $N_{\epsilon}$ is contained in the interval $[\epsilon, \infty)$. By theorem 1.3 we have that the essential spectrum of $\mathbf{L}$ is contained in $[\epsilon, \infty)$. Thus for any $\delta<\epsilon$, the number of eigenvalues of $\mathbf{L}$ smaller than $\delta$ is finite. In particular the index of $\mathbf{L}$ is finite. 


\section{Conformal Type of Minimal Surfaces}

It's well known that there exists no complete conformal metric $d s^{2}=\mathrm{e}^{2 u}|d z|$ on the complex plane $\mathbb{C}$, whose Gauss curvature satisfies $K \leq-1$. In this section we prove that any conformal metric on the complex plane, whose Gauss curvature is sufficiently negative outside a compact set must necessarily have non-negative total curvature. This will enable us to prove that the punctured disk $D^{*}=\{0<|z|<1\}$ can't be conformally immersed in $\mathbb{H}^{n}$ in such a manner that the immersion is a complete (at the origin) minimal surface.

Lemma 4.1. Let $d s^{2}=e^{2 u}|d z|$ be a conformal metric defined on $\mathbb{C}$. Suppose that the Gauss curvature satisfies $K(z) \leq-1 /|z|^{2}$ oustide a compact set $\Omega \subset$ C. Then $\int_{\mathbb{C}} K d A \geq 0$.

Proof. Let $(\rho, \theta)$ be the polar coordinates of $\mathbb{C}$ and let $d A=\mathrm{e}^{2 u} \rho d \rho d \theta$ be the area element for the metric $d s^{2}$. For $r>0$ we integrate both sides of the Gauss equation $\Delta u=-K \mathrm{e}^{2 u}$ over the disc $\{|z| \leq r\}$ to obtain

$$
\int_{|z| \leq r} \Delta u d x d y=-\int_{0}^{r} \int_{0}^{2 \pi} \mathrm{e}^{2 u} K \rho d \rho d \theta=-\int_{|z| \leq r} K d A .
$$

Let $I(r)=\frac{1}{2 \pi} \int_{0}^{2 \pi} u(r, \theta) d \theta$ and denote by $I^{\prime}(r)$ the derivative of $I(r)$. By the Green's formula and the above equation we get

$$
r I^{\prime}(r)=\frac{1}{2 \pi} \int_{0}^{2 \pi} \frac{\partial u}{\partial r}(r, \theta) r d \theta=-\frac{1}{2 \pi} \int_{0}^{r} \int_{0}^{2 \pi} \mathrm{e}^{2 u} K \rho d \rho d \theta .
$$

Taking derivatives with respect to $r$ gives

$$
\frac{1}{r}\left[r I^{\prime}(r)\right]^{\prime}=-\frac{1}{2 \pi} \int_{0}^{2 \pi} \mathrm{e}^{2 u} K d \theta .
$$

Let $r_{0}>0$ be such that $\Omega \subset\left\{|z|<r_{0}\right\}$. Then, for $|z| \geq r_{0}$ we have $K(z) \leq-1 /|z|^{2}$ and by Jensen's inequality we get

$$
r\left[r I^{\prime}(r)\right]^{\prime} \geq \frac{1}{2 \pi} \int_{0}^{2 \pi} \mathrm{e}^{2 u(r, \theta)} d \theta \geq \mathrm{e}^{\frac{1}{2 \pi} \int_{0}^{2 \pi} 2 u(r, \theta) d \theta}=\mathrm{e}^{2 I(r)} .
$$

Set $t=\ln r$, and $m(t)=I\left(\mathrm{e}^{t}\right)$. Denoting derivatives with respect to $t$ by a dot we have

$$
\dot{m}(t)=\left(\left(r I^{\prime}(r)\right)\left(\mathrm{e}^{t}\right) \quad \text { and } \quad \ddot{m}(t)=\left(r\left[r I^{\prime}(r)\right]^{\prime}\right)\left(\mathrm{e}^{t}\right)\right.
$$


From the above we get, with $t_{0}=\log r_{0}$,

$$
\begin{aligned}
& \dot{m}(t)=-\frac{1}{2 \pi} \int_{|z| \leq \mathrm{e}^{t}} K d A \\
& \ddot{m}(t) \geq \mathrm{e}^{2 m(t)} \quad \text { for } t \geq t_{0} .
\end{aligned}
$$

Suppose now that the conclusion of the lemma is not verified. From the above equations and the fact that the integral of $K$ over the disc $\left\{|z| \leq \mathrm{e}^{t}\right\}$ is a decreasing function of $t$ for $t$ large, there exists real numbers $a>0$ and $t_{1}>t_{0}$ such that $\dot{m}(t) \geq a$ for all $t \geq t_{1}$. Thus

$$
\frac{d}{d t}(\dot{m}(t))^{2}=2 \dot{m}(t) \ddot{m}(t) \geq 2 \dot{m}(t) \mathrm{e}^{2 m(t)}=\frac{d}{d t} \mathrm{e}^{2 m(t)} \quad ; \quad t \geq t_{1} .
$$

Integrating both sides from $t_{1}$ to $t \geq t_{1}$ we get

$$
\dot{m}^{2}(t) \geq \mathrm{e}^{2 m(t)}\left(1+c \mathrm{e}^{-2 m(t)}\right) \quad ; \quad c=\dot{m}^{2}\left(t_{1}\right)-\mathrm{e}^{2 m\left(t_{1}\right)} .
$$

Since $\dot{m}(t) \geq a$ for $t \geq t_{1}$ we have $m(t) \rightarrow \infty$ as $t \rightarrow \infty$. Take $t_{2}>t_{1}$ such that for $t \geq t_{2}$ we have $c \mathrm{e}^{-2 m(t)}>-\frac{1}{4}$. Thus for $t \geq t_{2}$ we obtain

$$
\dot{m}(t) \mathrm{e}^{-m(t)} \geq \frac{1}{2}
$$

and integrating both sides from $t_{2}$ to $t \geq t_{2}$ we get

$$
-\mathrm{e}^{-m(t)}+\mathrm{e}^{-m\left(t_{2}\right)} \geq \frac{1}{2}\left(t-t_{2}\right)
$$

But the left side of this inequality is bounded and the variable $t$ is supposed to be defined all over the reals. This contradiction establishes that for $t$ large enough we have $\int_{|z| \leq \mathrm{e}^{t}} K d A>0$, that proves the lemma.

For the sake of completeness we prove the following known lemma.

Lemma 4.2. Let $d s^{2}=e^{2 u}|d z|$ be a complete conformal metric defined on $\mathbb{C}$ such that the Gauss curvature $K$ satisfies $K \leq-1$ outside some compact set. Let $d(z)$ be the distance from the origin with respect to the metric ds ${ }^{2}$ and let $B(r)=\{z \mid d(z) \leq r\}$ be the geodesic ball of radius $r$. Let $L(r)$ denote the length of $\partial B(r)$. Then $L(r) \rightarrow 0$ as $r \rightarrow \infty$. 
Proof. By the precedent lemma $\int_{\mathbb{C}} K d A$ exists and is non-negative. As $K \leq-1$ outside a compact set, the total area of the complete surface $\left(\mathbb{C}, d s^{2}\right)$ is finite. A result of Huber [13, theorem 12] tells us that for a complete surface of finite total curvature and finite total area we have equality in the Cohn-Vossen inequality; thus

$$
\int_{\mathbb{C}} K d A=2 \pi \chi(\mathbb{C})=2 \pi
$$

where $\chi(\mathbb{C})$ is the Euler characteristic of the plane. For almost all $r$ the boundary $\partial B(r)$ is a finite union of piecewise differentiable Jordan curves and for those $r$ the derivative $L^{\prime}(r)$ exists and satisfies [18, theorem 1]

$$
L^{\prime}(r) \leq 2 \pi(2-2 h(r)-c(r))-\int_{B(r)} K d A
$$

where $c(r)=$ number of connected components of $\partial B(r)$ and $h(r)=$ number of handles inside $B(r)$. In our case $h(r)=0$ and $c(r) \geq 1$, so

$$
L^{\prime}(r) \leq 2 \pi-\int_{B(r)} K d A
$$

Let $r_{0}$ be such that for $r \geq r_{0}$ we have $K \leq-1$ on $\mathbb{C}^{*} \backslash B\left(r_{0}\right)$. Thus, for $r \geq r_{0}, \int_{B(r)} K d A$ is a decreasing function of $r$ which goes to $2 \pi$ as $r \rightarrow \infty$. Hence $L^{\prime}(r)<0$ for $r \geq r_{0}$. By the co-area formula we have

$$
\int_{0}^{\infty} L(r) d r \leq \int_{0}^{\infty}\left(\int_{\partial B(R)}|\nabla r|^{-1} d s\right) d r=\operatorname{Area}\left(\mathbb{C}, d s^{2}\right) .
$$

Therefore $\int_{0}^{\infty} L(r) d r<\infty$ and $L^{\prime}(r)<0$ for almost all $r \geq r_{0}$ and this implies that $L(r) \rightarrow 0$ as $r \rightarrow \infty$.

4.1. Proof of theorem B (conformal type). For $r>0$, we denote by $D^{*}(r)$ the punctured disc $\{0<|z|<r\}$ and we let $D^{*}$ be the unit punctured disc. The assertion about the conformal type of the ends of a minimal surface in hyperbolic space is a consequence of the following

Lemma 4.3. Let $x: D^{*} \hookrightarrow \mathbb{H}^{n}$ be a conformal minimal immersion. Then there exists a path $\gamma:[0,1) \rightarrow D^{*}$ converging to the origin 0 as $t \rightarrow 1$ and such that $\int_{\gamma} d s<\infty$, where $d s^{2}$ is the metric on $D^{*}$ induced by the immersion $x$. 
Proof. We consider the Poincaré model of $\mathbb{H}^{n}$ so that $\mathbb{H}^{n}$ is the unit ball $\{|x|<1\}$ of $\mathbb{R}^{n}$ endowed with the metric $d \eta^{2}=4|d x|^{2} /\left(1-|x|^{2}\right)^{2}$. The area element $d A$ of the metric $d s^{2}$ is given by

$$
d A=\frac{1}{2}\|\nabla x\|^{2} d u d v
$$

where $z=u+i v$ is a point of $\mathbb{C}$ and $\|\nabla x\|^{2}=\frac{4}{1-|x|^{2}}\left(\left|x_{u}\right|^{2}+\left|x_{v}\right|^{2}\right)$ is the hyperbolic norm of $\nabla x=\left(\nabla x^{1}, \ldots, \nabla x^{n}\right)$.

As $x$ is minimal we have by Gauss equation $K \leq-1$ on $D^{*}$. Extend the metric $d s^{2}$ to a smooth metric $d \tilde{s}^{2}$ on $\mathbb{C}^{*} \cup\{\infty\}$ such that inside $D^{*}\left(\frac{1}{2}\right)$ it coincides with $d s^{2}$. Let us suppose that the conclusion of the lemma does not hold. This means the metric $d \tilde{s}^{2}$ is a complete conformal metric on $\mathbb{C}^{*} \cup\{\infty\}$ satisfying $K \leq-1$ outside some compact set. By lemma 4.1 the total curvature is finite and in particular the area $\int_{D^{*}\left(\frac{1}{2}\right)} d A$ of $x\left(D^{*}\left(\frac{1}{2}\right)\right)$ is finite. Therefore

$$
\int_{D\left(\frac{1}{2}\right)}\|\nabla x\|^{2} d u d v<\infty
$$

Also, by the monotonicity theorem of Anderson [1], $x\left(D^{*}\left(\frac{1}{2}\right)\right)$ is contained in a compact set of $\mathbb{H}^{n}$; otherwise $x\left(D^{*}\left(\frac{1}{2}\right)\right)$ would have infinite area. This fact and (4.4) implies that the restriction of the immersion $x$ to $D^{*}\left(\frac{1}{2}\right)$ belongs to $H_{2}^{1}\left(D\left(\frac{1}{2}\right), \mathbb{H}^{n}\right)$, the space of maps $f: D\left(\frac{1}{2}\right) \mapsto \mathbb{H}^{n}$ such that $f$ and $|\nabla f|$ belong to $L_{2}\left(\left(D\left(\frac{1}{2}\right)\right)\right.$ (v. [10]).

On $D^{*}\left(\frac{1}{2}\right)$ the conformal minimal immersion $x$ satisfies the system of equations

$$
\Delta x^{i}=F^{i}(x, \nabla x) \quad ; \quad \text { for } i=1, \ldots, n
$$

where

$$
F^{i}(x, \nabla x)=\frac{2}{1-|x|^{2}}\left(x^{i}|\nabla x|^{2}-2\left\langle x, x_{u}\right\rangle x_{u}^{i}-2\left\langle x, x_{v}\right\rangle x_{v}^{i}\right) .
$$

We assert that $x$ is a weak solution of (4.5) on $D\left(\frac{1}{2}\right)$. In fact if $\phi=$ $\left(\phi^{1}, \ldots, \phi^{n}\right)$ is a smooth map compactly supported in $D\left(\frac{1}{2}\right)$ then the integrals

$$
I_{i}=\int_{D\left(\frac{1}{2}\right)}\left(\left\langle\nabla x^{i}, \nabla \phi^{i}\right\rangle+F^{i}(x, \nabla x) \phi^{i}\right) d u d v \quad ; \quad i=1, \ldots, n
$$


are well defined since $x$ is bounded in $D\left(\frac{1}{2}\right)$ and $x \in H_{2}^{1}\left(D\left(\frac{1}{2}\right), \mathbb{H}^{n}\right)$. Let $z_{0} \in \mathbb{C}^{*}$ be a fixed point and let $B(r)$ be the geodesic ball for the metric $d \tilde{s}^{2}$, of radius $r$ and centered in $z_{0}$. For $i=1, \ldots, n$ the integrals $I_{i}$ can be written as

$$
I_{i}=\lim _{r \rightarrow \infty} \int_{D^{*}\left(\frac{1}{2}\right) \cap B(r)}\left(\left\langle\nabla x^{i}, \nabla \phi^{i}\right\rangle+F^{i}(x, \nabla x) \phi^{i}\right) d u d v .
$$

Observe that for $r$ large enough the boundary $\partial B(r)$ is contained in $D\left(\frac{1}{2}\right)$ and that, by lemma 4.2 , the length of $\partial B(r)$ goes to 0 as $r \rightarrow \infty$. Let $\left\{r_{k}\right\}$, $k \in \mathbb{N}$, be a sequence with $r_{k} \rightarrow \infty$ as $k \rightarrow \infty$, and such that $\partial B\left(r_{k}\right)$ is a finite union of piecewise smooth curves. By equation (4.4) and Green's formula we get

$$
I_{i}=\lim _{k \rightarrow \infty} \int_{\partial B\left(r_{k}\right)} \phi^{i} \frac{\partial x^{i}}{\partial \nu}|d z|
$$

where $\nu$ is the interior normal to $\partial B\left(r_{k}\right)$, defined but for a finite number of points. Since $\left|\frac{\partial x^{i}}{\partial \nu}\right| \leq|\nabla x|$ we have that $\left|\frac{\partial x^{i}}{\partial \nu}\right||d z| \leq d s$ on $D^{*}\left(\frac{1}{2}\right)$. Hence

$$
\left|I_{i}\right| \leq\left(\max _{D\left(\frac{1}{2}\right)}|\phi|\right) \int_{\partial B\left(r_{k}\right)} d s .
$$

As the length of $\partial B\left(r_{k}\right)$ goes to 0 as $k \rightarrow \infty$ we have $I_{i}=0$ for $i=1, \ldots, n$, and therefore $x$ is a weak solution of (4.5) on $D\left(\frac{1}{2}\right)$. By the regularity result of Grüter [10, theorem 3.8] a minimal immersion $x$ as above is of class $C^{1, \alpha}$ on $D\left(\frac{1}{2}\right)$, for all $0<\alpha<1$. But this implies that any path $\gamma$ converging to the origin and having finite Euclidean length has also finite length in the induced metric $d s$. This contradiction establishes the lemma.

\section{REFERENCES}

1. Anderson, M. T., Complete Minimal Varietes in Hyperbolic Space, Invent. Math. 69 (1982) 477-494.

2. Gauss map, (preprint).

3. Chern, S. S. and Osserman, R., Complete minimal surfaces in $E^{n}$, J. d'Analyse Math. 19 (1967) 15-34.

4. Donnelly, H., On the essential spectrum of a complete Riemannian manifold, Topology 20 (1981) 1-14

5. Dodziuk, J., Vanishing theorems for square-integrable harmonic forms, Proc. Indian Acad. Sci. 90 (1981) 21-27.

6. Fischer-Colbrie, D., Complete minimal surfaces with finite Morse Index in threemanifolds, Invent. Math. 82 (1985) 121-132. 
7. Friedrichs, K. O., On the differentiability of solutions of linear elliptic differential equations, Comm. Pure and Appl. Math. 6 (1953) 299-326.

8. Gaffney, M. P., The Harmonic operator for exterior differential forms, Proc. National Acad. Sci., U.S.A 37 (1951) 48-50.

9. Glazman, I. M., Direct Methods of Qualitative Spectral Analysis of Singular Differential Operators, Daniel Davey, New York, 1965.

10. Grüter, M., Regularity of weak H-surfaces, J. reine angew. Math. 329 (1981) 1-15.

11. Hardt, R. and Lin, F. H., Regularity at infinity for area-minimizing hypersurfaces in hyperbolic space, Invent. Math. 88 (1987) 217-224.

12. Hoffman, D. and Spruck, J., Sobolev and isoperimetric inequalities for Riemannian submanifolds, Comm. Pure and Appl. Math. 27 (1974) 715-727.

13. Huber, A., On subharmonic functions and differential geometry in the large, Comment. Math. Helv. 32 (1957) 13-72.

14. Blaine Lawson, H., Lectures on minimal submanifolds, Publish or Perish, 1980.

15. Osserman, R., On Complete minimal surfaces, Arch. Rat. Mech. Anal. 13 (1963) 392-404.

16. Schoen, R., Estimates for stable minimal surfaces in three dimensional manifolds, Seminar on Minimal Submanifolds, Ann. of Math. Studies (1983) 111-126.

17. Simons, J., Minimal varietes in Riemannian manifolds, Ann. of Math. (2) 88 (1968) 62-105.

18. White, B., Complete surfaces of finite total curvature, J. Differential Geom. 26 (1987) 315-326.

Universidade Federal de Pernambuco, Brazil

E-mail address: oliveira@mathp7.jussieu.fr

ReCeIVEd April 8,1992 ReVISED OCTOBER 7,1992 